VESNA ELEZ

\title{
ARTICULER LES AFFECTS : FUIR DE JEAN-PHILIPPE TOUSSAINT
}

Depuis l'Antiquité, les affects occupent une place particulière dans la société et dans notre esprit. Puissants et redoutables pour Platon, ils fondent ses craintes relatives à l'art poétique. C'est précisément à eux que la poésie s'adresse, contournant la raison en tant que foyer stable de l'entendement humain. Leur nature irrationnelle suscite une profonde appréhension chez Platon à l'égard des conséquences morales que la représentation poétique peut entraîner.

En revanche, Aristote les considère cruciaux pour le fonctionnement de la catharsis. La pitié et la frayeur sont, à ses yeux, des affects indispensables pour que la catharsis s'opère : s'identifier avec le héros tragique par le biais de la frayeur et avoir de la pitié pour celui qui souffre injustement, voici les clés du bon entendement et du plaisir procuré par la représentation d'une tragédie. Les affects nous mènent vers leur propre transformation.

Depuis tant de siècles considérés comme un sujet à part entière par les philosophes tels que Platon, Aristote, Descartes, Pascal, Foucault, les affects, malgré la solidité de la raison, semblent gouverner nos vies d'une manière ou d'une autre, souvent contre notre gré. La place centrale dans ce domaine appartient aux relations amoureuses et à la manière dont nous essayons d'établir des liens durables avec d'autres personnes. Qu'en est-il avec l'homme contemporain ?

Appartenant aux écrivains des éditions de Minuit, Jean-Philippe Toussaint élabore sa stratégie narrative dans le sillage minimaliste de ses célèbres prédécesseurs, tels que les auteurs du Nouveau Roman, Robbe-Grillet, Simon ou bien Beckett. Pourtant, il ne s'agit pas uniquement de choisir un procédé peu commun, voire expérimental. Devenir auteur Minuit est une démarche à la fois esthétique et éthique ${ }^{1}$. Le hasard a voulu que le manuscrit de Toussaint, La Salle de bain, adressé à l'attention d'Alain Robbe-Grillet, soit lu par Jérôme Lindon, directeur des Éditions de Minuit, qui a aussitôt contacté l'écrivain en lui donnant

\footnotetext{
1 L'étude séminale d'Anne Simonin, Les Éditions de Minuit, 1942-1955. Le devoir de l'insoumission (Caen, IMEC Éditions, 1994), trace la première décennie et met en exergue les valeurs clés de cette maison d'édition pas comme les autres. Fondée en 1942 pendant l'occupation allemande, la maison a débuté en publiant Le silence de la mer de Vercors. Attachant une attention particulière aux auteurs novateurs, véritablement modernes, cet éditeur a également maintenu sa position morale et éthique, revendiquant la vérité et la liberté, notamment pendant la guerre d'Algérie. Malgré les changements et les crises au sein de la direction, après le départ de Pierre Lescure et Vercors, la maison continue à publier les ouvrages qui se distinguent par un procédé inédit sous la direction de Jérôme Lindon.
} 
une réponse favorable ${ }^{2}$. La Salle de bain, L'Appareil-photo, La Réticence sont les ouvrages qui l'ont classé parmi les meilleurs auteurs contemporains. Le style de la jeune génération de Minuit est dépouillé et épuré, doté d'une simplicité trompeuse.

Malgré le décor minimaliste ou banal, une écriture peu conventionnelle et imprévisible, les romans de Toussaint, d'après Sophie Berto, relèvent d'un fond métaphysique. Elle souligne son procédé postmoderne, qui consiste à mélanger les genres et les styles ${ }^{3}$. Plaçant les ouvrages de Jean Echenoz, Jean-Philippe Toussaint et Éric Chevillard sous l'égide du «roman ludique », Olivier BessardBanquy souligne aussi ce procédé éclectique où l'on mélange «les genres et les nombres, les discours et les idées ${ }^{4} »$. Cependant, le principe ludique, selon Bessard-Banquy, n'est pas une fin en soi, un simple exercice littéraire, mais la question du sens des choses, le rapport au monde ${ }^{5}$.

Dans le roman Fuir, qui fait partie de son « Cycle de Marie » (les trois autres étant Faire l'amour, La Vérité sur Marie et Nue), Jean-Philippe Toussaint dresse une dynamique des affects qui s'éloigne de l'écriture conventionnelle. Par un langage qui se prête au roman noir ou au roman policier, il tisse des fils narratifs qui ne se soumettent pas à un récit cohérent et prévisible.

Nous nous proposons de tracer la manière dont les affects s'articulent dans ce roman. Nous identifierons d'abord les figures facilement reconnaissables décrites par Roland Barthes dans ses Fragments d'un discours amoureux. Ensuite, nous inspecterons pourquoi l'amour fait mal. Finalement, nous examinerons la nature paradoxale des affects : les verbes de rupture et de distance ne font que confirmer la plénitude émotionnelle et la présence.

Doublement extirpé de son contexte d'origine, à la fois des autres volumes sur Marie et de son pays, le protagoniste-narrateur part en Chine pour remettre de la part de Marie une enveloppe remplie de milliers de dollars à un certain Zhang Xiangzhi, l'homme d'affaires douteux, à Shanghai. Il prend soin d'informer le lecteur que la rupture amoureuse avec Marie s'ensuit un an plus tard. Dans une ambiance cinématographique évoquée par un style désinvolte, lapidaire et pourtant précis, le narrateur raconte ses expériences. Le tourbillon de son séjour en Chine ne manque pas de se manifester. À l'instar d'un James Bond discret, troublé et légèrement distrait, toujours en mouvement, il fuit tout : le danger, l'amour, le crime, le bonheur, même la ville de Shanghai. La rencontre avec Li Qi, une belle Chinoise, offre la possibilité de tromper Marie et de tenter une nouvelle aventure. Cependant, au moment où il entame la séduction, le narrateur apprend la mort du

2 Voir Michèle Ammouche-Kremers, « Entretien avec Jean-Philippe Toussaint », in Michèle Ammouche-Kremers, Henk Hillenaar (éds.), Jeunes auteurs de Minuit, Amsterdam - Atlanta, Rodopi, 1994, p. 27.

${ }^{3}$ Sophie Berto, «Jean Philippe Toussaint et la métaphysique », in Michèle Ammouche-Kremers, Henk Hillenaar (éds.), Jeunes auteurs, pp. 18, 21.

${ }^{4}$ Olivier Bessard-Banquy, Le Roman ludique: Jean Echenoz, Jean-Philippe Toussaint, Éric Chevillard, Lille, Presses de Septentrion, 2003, p. 257.

${ }^{5}$ Ibidem. 
père de Marie et songe désormais à quitter la Chine afin d'assister à ses obsèques. Le problème qui se pose est la surveillance permanente à laquelle le narrateur se sent exposé : Zhang Xiangzhi possède un portable par lequel il communique avec Marie. En outre, le narrateur ne peut faire aucun geste sans qu'il ne soit tout de suite aperçu.

Il arrive ainsi à capter le mouvement, le déplacement et le décalage sans établir de véritables repères - sauf Marie, qui, malgré l'éloignement et la distance, reste le seul point fixe dans le périple des affects. Le titre du roman incarne le mouvement, le désir de s'éloigner. Passant néanmoins par de véritables non-lieux ${ }^{6}$, le hérosnarrateur esquisse la solitude et l'isolement de l'homme contemporain. Cependant, nous croyons que le titre révèle une autre signification, encore plus pertinente pour notre propos : le verbe fuir se réfère aussi à une suppression volontaire des affects, à un refoulement qui, à la fin, n'aboutira pas.

En traçant quelques lieux communs du discours amoureux proposé par Barthes, nous repérons ici les figures suivantes : L'absent, Exil, Insupportable, Loquèle, Nuit, Pleurer, Tendresse et peut-être Union. Barthes précise : «EXIL. Décidant de renoncer à l'état amoureux, le sujet se voit avec tristesse exilé de son Imaginaire ${ }^{7}$. $\gg$ Telle est la situation du narrateur :

Serait-il jamais fini avec Marie ? L'été précédant notre séparation, j'avais passé quelques semaines à Shanghai, ce n'était pas vraiment un déplacement professionnel, plutôt un voyage d'agrément, même si Marie m'avait confié une sorte de mission (mais je n'ai pas envie d'entrer dans les détails) ${ }^{8}$.

La solitude à Shanghai unit la figure de la nuit et celle de la solitude :

Accoudé au parapet, pensif, je regardais la surface noire et ondulante du fleuve dans l'obscurité, et je songeais à Marie avec cette mélancolie rêveuse que suscite la pensée de l'amour quand elle est jointe au spectacle des eaux noires dans la nuit'.

Cette scène établit d'emblée la ressemblance des émotions et des affects avec l'eau, le liquide, le fuyant, le puissant, le changeable, le profond. Barthes qualifie ces figures de la manière suivante : «NUIT. Tout état qui suscite chez le sujet la

\footnotetext{
${ }^{6}$ Voir Lidia Cotea, À la lisière de l'absence. L'imaginaire du corps chez Jean-Philippe Toussaint, Marie Redonnet et Éric Chevillard, Paris, L'Harmattan, 2013, p. 112 : « Pourtant, le plus souvent, ce déplacement extrême du corps n'a pas trop de consistance, le personnage s'arrêtant beaucoup dans des non-lieux comme le seraient les hôtels, les aéroports, les gares ou bien les cabines téléphoniques - façons de se perdre, de s'abandonner, de se fuir, de se glisser à dessein dans l'anonymat; de s'éprouver comme spectateur». Cotea ajoute que ces lieux, souvent vitrés, possèdent une double valeur, ils protègent le protagoniste du monde extérieur : «[...] autant d'endroits où le verre implique à la fois l'isolement et la protection par l'effet d'écran ».

${ }^{7}$ Roland Barthes, Fragments d'un discours amoureux, Paris, Seuil, 1977, p. 123.

${ }^{8}$ Jean-Philippe Toussaint, Fuir, Paris, Éditions de Minuit, 2005, p. 11.

${ }^{9}$ Ibidem, p. 20.
} 
métaphore de l'obscurité (affective, intellective, existentielle) dans laquelle il se débat ou s'apaise $»^{10}$.

La scène finale du roman est symétrique à celle-ci. Elle unit la figure d'étreinte, de la nuit, de la tendresse et de l'union. Son potentiel symbolique révèle la seule articulation adéquate de la nature des affects : le narrateur et Marie nagent à la fin de la journée, il part en quête effrénée da sa bien-aimée en la retrouvant avant la tombée de la nuit. L'eau, la dérive, la mer, représentent à la fois la profondeur des affects, leur nature changeante et imprévisible de la marée haute, l'incertitude sinistre et la proximité de la mort. Rappelant le lien intertextuel avec les romans de Camus ${ }^{11}$, la scène aquatique évoque les moments de bonheur dans L'Étranger ou dans La Peste: Mersault est heureux lorsqu'il se baigne avec Marie, Rieux et Tarrou nagent tranquillement dans la nuit. Chez Toussaint, il s'agit d'une scène archétypale, homérique, qui vient après une longue odyssée intérieure, la scène de retrouvailles, où les larmes rejoignent l'immensité émotionnelle et celle de la mer : « [...] je l'embrassais, je recueillais ses larmes avec les lèvres, je sentais l'eau salée sur ma langue, j'avais de l'eau de mer dans les yeux, et Marie pleurait dans mes bras, dans mes baisers, elle pleurait dans la mer $\rangle^{12}$.

Pourtant, avant de retrouver Marie et de l'embrasser, le narrateur passe par l'anticipation de leurs retrouvailles et le bonheur que cette idée lui procure, détruite aussitôt par l'angoisse et la panique, suivie par une quête fiévreuse de Marie. Cette partie du roman est un véritable tour de force qui s'oppose au romanesque, au conventionnel, qui contredit le lieu commun d'une rencontre heureuse où les amants se rejoignent et les affects se transforment en bonheur. À l'encontre des présupposés du lecteur, Toussaint opte pour une spirale asphyxiante où les contretemps, l'absence et les retards soulignent la nature contradictoire des affects qui atteignent leur plénitude non dans une étreinte synchronisée, mais dans la perte et dans l'absence. C'est une manière de briser le 《 pacte romanesque $»^{13} \mathrm{du}$ lecteur, de lui véhiculer l'aspect douloureux du sentiment amoureux.

Y-a-t-il un parti pris de la faute aussi ? Dans la figure des Fautes, Barthes explique : «FAUTES. En telle ou telle occasion infime de la vie quotidienne, le sujet croit avoir manqué à l'être aimé et en éprouve un sentiment de culpabilité. » Barthes rappelle les propos de Nietzsche et croit pouvoir affranchir la douleur de

\footnotetext{
${ }^{10}$ Roland Barthes, Fragments, p. 203.

11 Toussaint évoque L'Étranger en parlant de l'un de ses romans. Pourtant, il souhaite créer un héros plus décontracté et moins sérieux : «Il y a certes une proximité thématique mais je voulais m'éloigner tout à fais du sérieux de L'étranger, qui, par ailleurs, continue à me fasciner ; ce livre a une force d'évidence qui me fait parfois penser à L'amant de Duras, une sobriété de moyens, c'est minimaliste quoi !... (rires)» (Michèle Ammouche-Kremers, « Entretien », p. 34).

12 Jean-Philippe Toussaint, Fuir, p. 186.

${ }^{13}$ Voir Dominique Viart, Le roman français au XXe siècle, Paris, Hachette supérieur, 1999, p. 140 : «Au lieu d'exploiter les ressorts romanesques de toutes les façons possibles, Jean-Philippe Toussaint préfère jouer de leur absence. Il s'amuse des attentes d'un lecteur rompu au 'pacte romanesque', en créant des situations d'attente pour mieux les décevoir ».
} 
l'idée de faute, de rendre à la douleur son innocence, l'innocence de la passion : «non pas du tout une pureté, mais tout simplement le rejet de la Faute» ${ }^{14}$. Même pendant leurs retrouvailles à Elbe, le narrateur n'arrive pas à s'épanouir harmonieusement dans cette relation: «Le responsable de ses souffrances, ce devint moi, moi qui la tourmentais même en ne faisant rien - ma simple présence la faisait souffrir, et mon absence encore plus -, moi qui n'avais pas été là quand elle avait eu besoin de moi $»^{15}$.

La figure de l'Absence est aussi en jeu : «ABSENCE. Tout épisode de langage qui met en scène l'absence de l'objet aimé - quelles qu'en soient la cause et la durée - et tend à transformer cette absence en épreuve d'abandon ${ }^{16}$. »

La tentative d'aventure avec la belle Chinoise Li Qi s'inscrit dans le champ de la figure Étreinte, où un nouveau corps tente de remplacer celui de Marie. Plusieurs références ultérieures mettront en évidence la ressemblance explicite des gestes de Li Qi avec celles de Marie. Li Qi n'est qu'un double de Marie dont la présence ne fait que rappeler celle de Marie. L'étreinte finale avec Marie effacera entièrement la brévité de celle avec Li Qi.

Lorsqu'il apprend la mort du père de Marie et après avoir entendu sa voix au téléphone, le narrateur abandonne brusquement Li Qi est se livre à la figure de Pleurer: "PLEURER. Propension particulière du sujet amoureux à pleurer: modes d'apparition et fonction des larmes chez ce sujet ${ }^{17}$. " Il est à noter que, quoique chagriné par la triste nouvelle que le narrateur vient d'apprendre, il est aussi bouleversé par les affects accumulés et difficile à dissocier : l'amour pour Marie, et par extension pour son père, la douleur ressentie pour la souffrance de l'être aimé, sa propre douleur et ses propres troubles amoureux :

Je me sentais submergé par l'envie de pleurer, et je me raccrochais à cette voix douce qui me berçait $[\ldots]$

Je pleurais. J'étais debout dans le train, et je pleurais en silence, sans humeurs et sans larmes, le front en sueur et ma chemise défaite ${ }^{18}$.

Cette figure rejoint celle de la Loquèle qui reflète le comportement du protagoniste. Les pensées qui se succèdent dans son esprit se conforment à la description de Barthes :

LOQUÈLE. Ce mot, emprunté à Ignace de Loyola, désigne le flux de paroles à travers lequel le sujet argumente inlassablement dans sa tete les effets d'une blessure ou les conséquences d'une conduite : forme emphatique du 'discourir' amoureux ${ }^{19}$.

\footnotetext{
${ }^{14}$ Roland Barthes, Fragments d'un discours amoureux, p. 136.

${ }^{15}$ Jean-Philippe Toussaint, Fuir, p. 156.

${ }^{16}$ Roland Barthes, Fragments d'un discours amoureux, p. 19.

${ }^{17}$ Roland Barthes, Fragments d'un discours amoureux, p. 213.

${ }^{18}$ Jean-Philippe Toussaint, Fuir, p. 52.

${ }^{19}$ Roland Barthes, Fragments d'un discours amoureux, p. 191.
} 
Le narrateur est conscient que la voix de Marie est la métonymie de tout son univers, du passé qui s'avère être le présent par cet asyndète animé :

La faible voix de Marie qui me transportait littéralement, comme peut le faire la pensée, le rêve ou la lecture, quand, dissociant le corps de l'esprit, le corps reste statique et l'esprit voyage [...] derrière nos yeux fermés, naissent des images et resurgissent des souvenirs, des sentiments et des états nerveux, se ravivent des douleurs, des émotions enfouies, des peurs, des joies, des sensations, de froid, de chaud, d'être aimé, de ne pas savoir, dans un afflux régulier de sang dans les tempes, une accélération régulière des battements du cœur, et un ébranlement, comme une lézarde, dans la mer de larmes séchées qui est gelée en nous ${ }^{20}$.

L'ultime phrase du roman transmet le goût salé des larmes de Marie qui remontent à la surface après être étouffées auparavant, comme une catharsis désirée, comme une libération affective, mêlée à ses pulsions combatives, en guise de soulagement : « [...] elle attendit le dernier mètre, elle attendit d'arriver à ma hauteur et de poser la main sur mon épaule pour fondre en larmes, m'embrassant et me frappant tout à la fois $»^{21}$.

Pourquoi était-il difficile de rester avec Marie malgré l'amour profondément ressenti ? Barthes évoque la figure de l'Insupportable, une variante de should I stay or should I go: "INSUPPORTABLE. Le sentiment d'une accumulation des souffrances amoureuses explose dans ce cri : "Ça ne peut pas continuer" $\gg$ ". Le lecteur devine qu'avant la rupture officielle, l'amour-passion et ses complications étouffaient le narrateur tout en approfondissant ses émotions. Ce paradoxe, cet oxymore affectif est souvent à l'origine de la souffrance amoureuse. Le narrateur est conscient qu'il n'y échappera pas, malgré sa fuite.

Olivier Bessard-Banquy souligne à juste titre la position paradoxale du héros face aux affects, il met en valeur «l'intrication de la logique narcissique et de l'ordre amoureux »: " [...] plus le héros est à la recherche de lui-même et plus il est en demande d'affects ou d'amour et plus il dépend potentiellement d'autrui et plus son existence se réduit à une quête amoureuse $»^{23}$. Il conclut que « la 'jeune' littérature de Minuit, parmi d'autres, souligne ainsi la difficulté d'aimer », tout en soulignant l'importance de l'articulation littéraire des affects :

Ici encore la littérature apparaît plus que nécessaire ; non seulement, par les mots, elle nourrit l'imaginaire du désir, mais en donnant voix à différentes manières d'aimer, elle crée chez son lecteur une sorte d'écho et l'aide à mieux se connaître, à mieux se comprendre, à mieux se penser ${ }^{24}$.

\footnotetext{
${ }^{20}$ Jean-Philippe Toussaint, Fuir, pp. 51-52.

${ }^{21}$ Ibidem, p. 185.

22 Roland Barthes, Fragments, p. 167.

${ }^{23}$ Olivier Bessard-Banquy, "La relation amoureuse chez les 'jeunes auteurs de Minuit' », in Marc Dambre, Bruno Blanckeman (éds.), Romanciers minimalistes 1979-2003, Paris, Presses Sorbonne Nouvelle, 2012, consulté le 11 novembre 2018, https://books.openedition.org/psn/431.

${ }^{24}$ Ibidem.
} 
Le corps, en tant que foyer affectif, reflète cette absence d'harmonie et d'entente naturelle entre les amants. Lidia Cotea soutient que cette négativité se manifeste par « une série de gestes et attitudes contradictoires qui indiquent, malgré l'atmosphère d'intimité, qu'il leur est impossible de s'aimer et qui mènent à une séparation des partenaires $»^{25}$. Certes, mais le déséquilibre entre les amants représente, à notre avis, une autre attaque discrète contre le romanesque dans ce roman de Toussaint: le bonheur et la plénitude réalisés lors d'une rencontre amoureuse représentent souvent un présupposé idéalisé - les affects se nourrissent par l'absence et par le manque, ils sont rarement synchronisés ou parfaitement réciproques ${ }^{26}$.

Barthes illustre ces épreuves par la résistance aux coups du destin :

Je subis sans m'accommoder, je persiste sans m'aguerrir: je suis une poupée Daruma, un poussah sans jambes auquel on donne des chiquenaudes incessantes, mais qui finalement reprend son aplomb, assuré par une quille intérieure (mais quelle est ma quille? La force de l'amour ? ${ }^{27}$.

La quille rappelle une des scènes du roman où le narrateur se rend dans une salle de bowling à Pékin, accompagné de Li Qi et de Zhang. Il commence mal, d'une main tremblante, mais gagne bientôt en sécurité et fait tomber presque toutes les quilles. Le bowling est une métaphore de la fragilité et de l'instabilité de la vie même, ainsi que des réactions fortuites et d'un savoir-faire intuitif qui est le seul en mesure de mettre fin à une crise amoureuse.

Pourquoi est-il si difficile de maintenir une relation amoureuse où les problèmes, malgré l'amour fort entre les partenaires, semblent l'emporter sur le bonheur du couple ? Pourquoi l'amour fait-il mal, se demande Eva Illouz dans son étude éponyme. Son argument implique un changement décisif dans le comportement affectif après le romantisme. Nous vivons nos peines d'amour différemment, et cette transformation concerne trois catégories fondamentales de notre personnalité, d'après Illouz. Elles sont les suivantes : la volonté, à savoir la manière dont on veut quelque chose ; la reconnaissance, ce qui est important pour notre sens de la valeur, et finalement le désir, ce dont on a envie et la manière dont on éprouve ce désir. Bien que la peine d'amour soit transformée, nous ne sommes pas épargnés de l'agonie amoureuse : notre quête de l'amour est une expérience extrêmement difficile ${ }^{28}$. Pour elle, malgré le nombre croissant des livres qui visent à reconnaître certaines matrices qui sont à l'origine de notre mal, le problème ne

\footnotetext{
${ }^{25}$ Lidia Cotea, À la lisière de l'absence, p. 148.

${ }^{26}$ Bruno Blanckeman rappelle que déjà dans le roman Faire l'amour « l'union physique est décrite comme une suite de symptômes tannatiques » (Bruno Blanckeman, "Faire l'amour à la Toussaint (sur quelques postures littéraires minimalistes) », in Marc Dambre, Bruno Blanckeman (éds.), Romanciers minimalistes 1979-2003, Paris, Presses Sorbonne Nouvelle, 2012, consulté le 11 novembre 2018, https://books.openedition.org/psn/430).

${ }^{27}$ Roland Barthes, Fragments, p. 168.

${ }^{28}$ Eva Illouz, Why Love Hurts, Cambridge, Polity Press, 2012, p. 3.
} 
réside pas dans une enfance dysfonctionnelle ni dans les psychés immatures : ce qui cause le trouble, c'est l'ensemble des tensions sociales et culturelles qui structurent le «moi » moderne et notre identité ${ }^{29}$. L'importance de l'amour en tant que catégorie clé de notre bonheur et de notre identité va de pair avec la difficulté de cet aspect de notre expérience : les deux touchent à la manière dont le « moi » et l'identité sont institutionnalisés dans la modernité ${ }^{30}$. Illouz rappelle que les défis de la vie moderne ne sont pas négligeables, puisqu'ils réduisent notre capacité d'épanouissement. Dans les chapitres ultérieurs de son étude, elle examine la peur de l'engagement, la vulnérabilité, les différents besoins individuels d'être reconnu et aimé. Cependant, elle affirme que c'est la révolution moderne de l'amour hétérosexuel qui a changé le paradigme de l'amour: les relations sexuelles se sont affranchies des normes morales. C'est la prépondérance des modèles économiques qui façonnent désormais le « moi » et ses émotions ${ }^{31}$.

Les analyses d'Ilouz s'appliquent bien à la relation complexe entre le narrateur et Marie dans le roman Fuir. De toute évidence, la pertinence de la question d'argent s'impose dès le début du récit : le protagoniste se dirige à Shanghai afin de remettre un grand montant d'argent de la part de Marie. Elle est issue d'un milieu très aisé - le lecteur, même s'il n'avait appris dans les volumes antérieurs que Marie est une designer de mode, peut évaluer son statut par la richesse de son père. Il possède une maison à l'île d'Elbe et, malgré sa désinvolture et un certain isolement social, il fait partie d'un milieu social privilégié.

En outre, les rôles traditionnels du masculin et du féminin se trouvent inversés ici. D'après Illouz, les hommes dominent les règles de reconnaissance et d'engagement - la domination masculine devient un modèle d'autonomie auquel les femmes aspirent dans leur lutte d'égalité dans leur vie publique. Néanmoins, une fois transférée dans la sphère privée, l'égalité trouble les femmes et étouffe leur besoin de reconnaissance ${ }^{32}$. Dans le roman, Marie est porteuse des qualités proprement masculines : elle sait ce qu'elle veut, elle s'obstine, elle est même parfois une femme qui domine impérieusement: vêtue en cavalière pour les obsèques de son père, elle dirige tout, tout est soumis à sa volonté :

Marie était seule en face du cercueil, droite dans une chemise blanche et un pantalon beige strictement ceinturé, le regard dur, froid, sombre, avec quelque chose de buté dans l'attitude. Quand elle me vit, me reconnut, elle me dévisagea avec détresse, une bouffée de douleur envahit son visage, mais elle retrouva aussitôt son sang-froid, et redevint froide, digne, distante, elle me fit simplement signe de la main d'aller m'asseoir à l'écart sur un banc, mais pas à côté d'elle, elle ne me dit pas de la rejoindre ${ }^{33}$.

\footnotetext{
${ }^{29}$ Ibidem, p. 4.

${ }^{30}$ Ibidem, p. 6.

${ }^{31}$ Ibidem, p. 9.

${ }^{32}$ Eva Illouz, Why Love Hurts, p. 137.

${ }^{33}$ Jean-Philippe Toussaint, Fuir, p. 146.
} 
Marie, une femme qui se veut autonome, est fragile et, par-dessus tout, fragilisée à l'extrême par la mort de son père. Le narrateur le sait, le sent, et malgré l'amour qui comprend toute sorte de sacrifice et d'empathie profonde, il revendique en même temps que Marie reconnaisse sa vulnérabilité aussi. Le rôle traditionnel de l'émotivité féminine appartient ici plutôt au narrateur qu'à Marie. Barthes dresse le portrait de ce type d'homme en décrivant de près la figure de l'Absent. Puisque, traditionnellement, c'est la femme qui attend l'homme qui est partie à la chasse, elle est fidèle, sédentaire, d'après Barthes :

...il s'ensuit que dans tout homme qui parle de l'absence de l'autre, du féminin se déclare : cet homme qui attend et qui en souffre, est miraculeusement féminisé. Un homme n'est pas féminisé parce qu'il est inverti, mais parce qu'il est amoureux ${ }^{34}$.

Apparemment, la synchronicité de la reconnaissance de la fragilité mutuelle n'a pas eu lieu. Toujours en décalage, le statut du couple et leurs affects se confirment paradoxalement par les verbes d'absence, de distance, d'inaccessibilité : partir, fuir, sortir, se séparer, se déplacer, s'éloigner, quitter, disparaître, voyager, courir, se cacher, prendre l'avion, prendre le train, etc. Les deux tiers du récit se déroulent en l'absence de Marie, si bien que cette stratégie révèle sa présence indirecte et écrasante.

Le procédé narratif en soi transmet symboliquement la véritable nature des affects : l'incertitude et le dénouement de la trame du roman policier, de la fuite et la course avec Zhang et Li Qi sur la moto, l'absence des réponses à l'intrigue policière : devant qui fuient-ils, pourquoi, quel est le contenu du colis que Li Qi avait transmis à Zhang, quel est le véritable rapport entre Li Qi et Zhang, etc. Donc, aucune certitude, aucune affirmation. Les affects sont non-linéaires, récalcitrants, susceptibles de réapparaître aux moments inattendus. Une réitération non anticipée semble les caractériser, malgré le constat qu'ils ne se soumettent à aucune loi. Même les retrouvailles à l'île d'Elbe s'articulent par les va-et-vient successifs, par le croisement, par la fougue, par des malentendus, par la quête qui devient effrénée vers la fin du roman, dans la scène de la baignade dans la mer, où les affects cherchent à s'épanouir.

Pour conclure, remarquons que le roman mûr de Jean-Philippe Toussaint dépasse, à notre avis, les acquis de sa prose antérieure. Par-delà un procédé ludique, le roman Fuir est rythmé par la nature même des affects, qui se cachent et se perdent afin de mieux ressurgir ou d'exploser. S'il privilégie le récit sur la diégèse ${ }^{35}$ dans ses précédents ouvrages, ici il soumet le procédé narratif à la logique non-linéaire des affects, il suit leur articulation. Toussaint respecte les parties intégrantes de l'intrigue amoureuse (la présence des figures barthésiennes le

\footnotetext{
${ }^{34}$ Roland Barthes, Fragments, p. 20.

${ }^{35}$ Voir Dominique Viart, Le roman français, p. 141 : «Contrairement aux raconteurs d'histoires, ces romanciers privilégient le récit sur la diégèse. L'histoire importe peu : l'élan narratif l'emporte et marque une certaine indifférence envers les contraintes purement diégétiques ».
} 
confirme) en les abordant autrement, évitant le conventionnel et le romanesque. Le roman Fuir semble incarner la quintessence de la fameuse "impassibilité », la valeur phare des écrivains de Minuit, que Jérôme Lindon définit ainsi: «[...] ça n'est pas l'équivalent d'insensible qui n'éprouve rien ; cela signifie précisément le contraire : qu'on ne trahit pas ses émotions $»^{36}$.

\section{BIBLIOGRAPHIE}

AMMOUCHE-KREMERS, Michèle, «Entretien avec Jean-Philippe Toussaint », in Michèle Ammouche-Kremers, Henk Hillenaar (éds.), Jeunes auteurs de Minuit, Amsterdam - Atlanta, Rodopi, 1994, pp. 27-35.

BARTHES, Roland, Fragments d'un discours amoureux, Paris, Seuil, 1977.

BERTO, Sophie, «Jean Philippe Toussaint et la métaphysique », in Michèle Ammouche-Kremers, Henk Hillenaar (éds.), Jeunes auteurs de Minuit, Amsterdam - Atlanta, Rodopi, 1994, pp. 1525.

BESSARD-BANQUY, Olivier, "La relation amoureuse chez les 'jeunes auteurs de Minuit' », in Marc Dambre, Bruno Blanckeman (éds.), Romanciers minimalistes 1979-2003, Paris, Presses de Sorbonne Nouvelle, 2012, pp. 155-164, consulté le 11 novembre, https://books.openedition.org/psn/431.

BESSARD-BANQUY, Olivier, Le Roman ludique: Jean Echenoz, Jean-Philippe Toussaint, Éric Chevillard, Lille, Presses Universitaires du Septentrion, 2003.

BLANCKEMAN, Bruno, "Faire l'amour à la Toussaint (sur quelques postures littéraires minimalistes) », in Marc Dambre, Bruno Blanckeman (éds.), Romanciers minimalistes 1979_ 2003, Paris, Presses Sorbonne Nouvelle, 2012, pp. 141-153, consulté le 11 novembre 2018, https://books.openedition.org/psn/430.

COTEA, Lidia, À la lisière de l'absence. L'imaginaire du corps chez Jean-Philippe Toussaint, Marie Redonnet et Éric Chevillard, Paris, L'Harmattan, 2013.

ILOUZ, Eva, Why Love Hurts, Cambridge, Polity Press, 2012.

LINDON Jérôme, Jean-Pierre Salgas, "Jérôme Lindon: On ne se baigne pas deux fois dans le même fleuve ", La Quinzaine littéraire, 1989, 532, p. 34.

SIMONIN, Anne, Les Éditions de Minuit 1942-1955. Le Devoir d'insoumission, Caen, IMEC Éditions, 1994.

TOUSSAINT, Jean-Philippe, Fuir, Paris, Éditions de Minuit, 2005.

VIART, Dominique, Le roman français au XXe siècle, Paris, Hachette supérieur, 1999.

\section{ARTICULATING THE AFFECTS: JEAN-PHILIPPE TOUSSAINT'S RUNNING AWAY (Abstract)}

In his novel Running Away (a part of his "Cycle of Marie", the other three being Making Love, The Truth about Marie and Naked), Jean-Philippe Toussaint offers an unconventional narrative

\footnotetext{
36 Jérome Lindon et Jean-Pierre Salgas, « Jérôme Lindon : On ne se baigne pas deux fois dans le même fleuve », La Quinzaine littéraire, 1989, 532, p. 34.
} 
treatment of the affective dynamics. The narrator-protagonist informs the reader that the novel is about his attempted separation from Marie. He leaves to China. The plot comprises several episodes which are not directly linked to the affects, offering a roman noir pattern of sorts. However, the affective canvas is overwhelming: although hardly articulated, the affects inundate the text by their silent presence. We intend to demonstrate that by using the verbs of movement which create distance between each other (leave, run, move, abandon, disappear, etc.) the narrator underlines Marie's omnipresence, although she actually appears in the last section of the novel. Her absence translated into her presence articulates the paradoxical nature of the affects which cannot be dominated by a rational decision and which defy the very narrative's linearity. Following Roland Barthes' arguments in his essay A Lovers' Discourse: Fragments, we aim to underline the paradoxical narrative strategy which confirms the predominance of the affects using the imagery of emptiness, void and escape.

Keywords: Jean-Philippe Toussaint, Fuir, Roland Barthes, affects, roman noir.

\section{ARMONIZAREA EMOȚIILOR: $S \breve{A} F U G I !$ de JEAN-PHILIPPE TOUSSAINT (Rezumat)}

În romanul Să fugi! (din Ciclul Mariei, care mai cuprinde romanele Să faci dragoste, Adevărul despre Mary şi Camera de baie) Jean-Philippe Toussaint oferă o abordare neconvenţională a dinamicii afectelor. Protagonistul-narator îi mărturiseşte cititorului că romanul este despre încercarea sa de a se despărţi de Marie. De aceea, pleacă în China. Acţiunea cuprinde o serie de episoade care nu sunt direct legate de afecte, prin formula unui roman noir. $\mathrm{Cu}$ toate acestea, structura emoţională este precumpănitoare: fie şi vag exprimate, sentimentele inundă textul printr-o prezenţă tăcută. Intenţia noastră este de a demonstra că, folosind verbele de mişcare ce creează distanţa (a pleca, a fugi, a se mişca, a abandona, a dispărea etc.), naratorul subliniază omniprezenţa Mariei, chiar dacă aceasta apare în realitate doar în ultima parte a romanului. Absenţa ei tradusă prin prezenţă relevă natura paradoxală a afectelor, care nu pot fi dominate printr-o decizie raţională, întrerupând însuşi caracterul linear al expunerii. Urmând ideile lui Roland Barthes din eseul Discursul îndrăgostit: Fragmente, dorim să scoatem în evidenţă strategia narativă paradoxală ce confirmă rolul predominant al sentimentelor, prin intermediul unor imagini precum golul, vidul şi evaziunea.

Cuvinte-cheie: Jean-Philippe Toussaint, Fuir, Roland Barthes, afecte, roman noir. 